\title{
The anti-IgE mAb omalizumab induces adverse reactions by engaging Fc $\gamma$ receptors
}

\author{
Bianca Balbino, ${ }^{1,2}$ Pauline Herviou, ${ }^{1}$ Ophélie Godon, ${ }^{1}$ Julien Stackowicz, ${ }^{1,2}$ Odile Richard-Le Goff,' Bruno lannascoli, ${ }^{1}$ \\ Delphine Sterlin, ${ }^{1,3}$ Sébastien Brûlé, ${ }^{4}$ Gael A. Millot, ${ }^{5}$ Faith M. Harris, ${ }^{6}$ Vera A. Voronina, ${ }^{6}$ Kari C. Nadeau, ${ }^{7,8}$ Lynn E. Macdonald, ${ }^{6}$ \\ Andrew J. Murphy, ${ }^{6}$ Pierre Bruhns, ${ }^{1}$ and Laurent L. Reber ${ }^{1,9}$ \\ 'Unit of Antibodies in Therapy and Pathology, Institut Pasteur, UMR1222 INSERM, Paris, France. ${ }^{2}$ Sorbonne Université, Paris, France. ${ }^{3}$ Assistance Publique-Hôpitaux de Paris, La Pitié-Salpêtrière, \\ Département d'Immunologie, Paris, France. ${ }^{4}$ Plateforme de Biophysique Moléculaire, Institut Pasteur, UMR 3528 CNRS, Paris, France. ${ }^{5}$ Hub de Bioinformatique et Biostatistique, Département Biologie \\ Computationnelle, Institut Pasteur, USR 3756 CNRS, Paris, France. ${ }^{6}$ Regeneron Pharmaceuticals Inc., Tarrytown, New York, USA. ${ }^{7}$ Sean N. Parker Center for Allergy and Asthma Research, Stanford University, \\ Stanford, California, USA. ${ }^{8}$ Division of Pulmonary and Critical Care, Department of Medicine, Stanford University, California, USA. ${ }^{9}$ Center for Physiopathology of Toulouse-Purpan (CPTP), UMR 1043, \\ University of Toulouse, INSERM CNRS, Toulouse France.
}

\begin{abstract}
Omalizumab is an anti-IgE monoclonal antibody ( $\mathrm{mAb}$ ) approved for the treatment of severe asthma and chronic spontaneous urticaria. Use of omalizumab is associated with reported side effects ranging from local skin inflammation at the injection site to systemic anaphylaxis. To date, the mechanisms through which omalizumab induces adverse reactions are still unknown. Here, we demonstrated that immune complexes formed between omalizumab and IgE can induce both skin inflammation and anaphylaxis through engagement of IgC receptors (FcyRs) in FcyR-humanized mice. We further developed an Fc-engineered mutant version of omalizumab, and demonstrated that this mAb is equally potent as omalizumab at blocking IgE-mediated allergic reactions, but does not induce Fc $\gamma \mathrm{R}$-dependent adverse reactions. Overall, our data indicate that omalizumab can induce skin inflammation and anaphylaxis by engaging FcyRs, and demonstrate that Fc-engineered versions of the mAb could be used to reduce such adverse reactions.
\end{abstract}

\section{Introduction}

IgE antibodies (Abs) are key mediators of allergic diseases (1-3). Upon exposure to an allergen in allergic patients, such allergen is recognized by IgE bound to the high-affinity receptor FceRI on the surface of mast cells and basophils, which promotes the immediate activation of these cells and the release of inflammatory mediators such as histamine, responsible for allergic symptoms (3).

Omalizumab (Xolair) is a recombinant humanized IgG1 mAb directed against IgE (4). Omalizumab binds to the C 83 domain of free IgE, and thereby impairs binding of IgE to both FceRI and the low-affinity IgE receptor CD23 (FceRII) (5-7). Omalizumab does not recognize IgE already bound to FceRI or CD23, and therefore cannot induce cell activation by crosslinking of IgE receptors (5, 7). Omalizumab is approved for the treatment of severe asthma (8) and chronic spontaneous urticaria (9). It also shows promise for the treatment of other allergic diseases, including food allergy (10).

However, treatment with omalizumab is associated with adverse reactions, ranging from skin inflammation at the injection

Authorship note: PB and LLR contributed equally to this work. Conflict of interest: $\mathrm{BB}, \mathrm{PB}$, and LLR are inventors on a patent related to this work (PCT/EP2019/059414). LEM and AJM are employees of Regeneron Pharmaceuticals, Inc., hold stock in the company, and are inventors on patents and patent applications related to the mice used for this work (US 8,658,154; US 8,883,496; US 9,221,984; US $9,687,566$; US 10,426,848; US 9,474,255; US 8,658,853). PB is a paid consultant for Regeneron Pharmaceuticals.

Copyright: @ 2020, American Society for Clinical Investigation.

Submitted: April 22, 2019; Accepted: November 13, 2019; Published: January 27, 2020 Reference information: J Clin Invest. 2020;130(3):1330-1335.

https://doi.org/10.1172/JCI129697. site to anaphylaxis $(\sim 0.1 \%-0.2 \%$ of patients) $(11-13)$. The mechanism of these side effects is still unknown. Notably, omalizumab does not induce the formation of anti-drug Abs, and most cases of anaphylaxis occur within the first 3 injections of the drug (11-13).

We hypothesized that the formation of immune complexes (ICs) between omalizumab and IgE could be responsible for some of the adverse reactions observed with this therapeutic mAb. Using mice humanized for all IgG receptors (Fc $\gamma \mathrm{Rs}$ ), we demonstrate here that omalizumab/IgE ICs can induce skin inflammation at the site of injection of the drug as well as systemic anaphylaxis through engagement of Fc $\gamma$ Rs. Finally, we developed an Fc-engineered version of omalizumab that blocks IgE-mediated allergic reactions without inducing Fc $\gamma \mathrm{R}$-dependent adverse reactions.

\section{Results and Discussion}

We first coincubated omalizumab and human $\operatorname{IgE}$ (termed $\operatorname{IgE}$ herein) in vitro to form ICs, and assessed the molecular mass of these ICs by size exclusion chromatography coupled to static light scattering (SEC-SLS). As reported previously $(14,15)$, these ICs were of limited size, mainly consisting of trimeric structures (Supplemental Figure 1; supplemental material available online with this article; https://doi.org/10.1172/JCI129697DS1). It was initially suggested that such small ICs have a low potential to engage Fc $\gamma$ Rs (15). However, we found that these ICs potently bind all activating human Fc $\gamma$ Rs (Fc $\gamma$ RI, IIA, IIIA, and IIIB), but not the inhibitory Fc $\gamma$ RIIB that has the lowest affinity for human IgG1 among Fc $\gamma$ Rs (16) (Figure 1A). As expected, we also observed that omalizumab binds human complement component $\mathrm{C} 1 \mathrm{q}$ in a dose-dependent manner (Figure 1B). 


\section{$\mathbf{A}$}
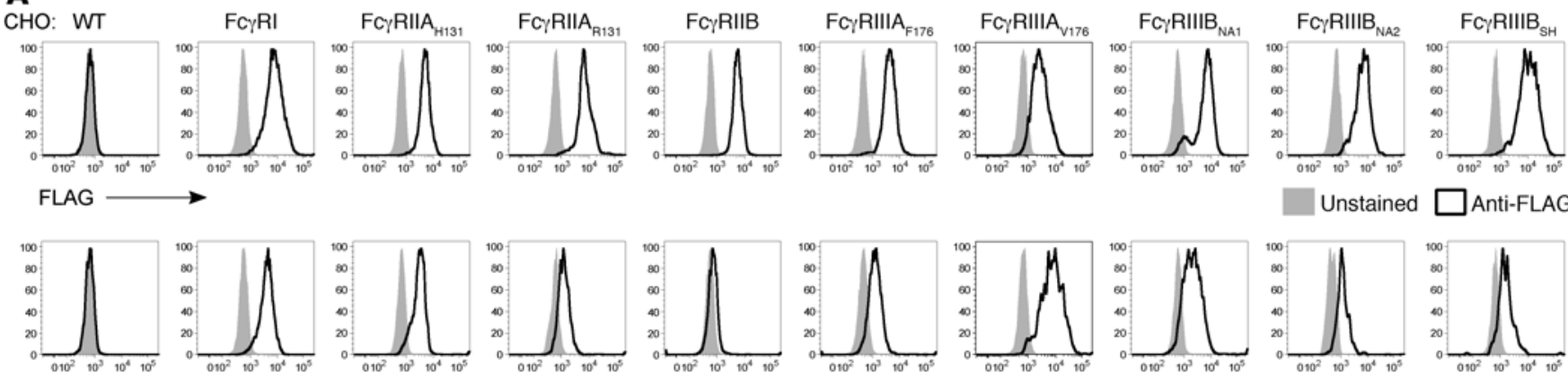

Unstained

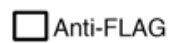

FITC

B

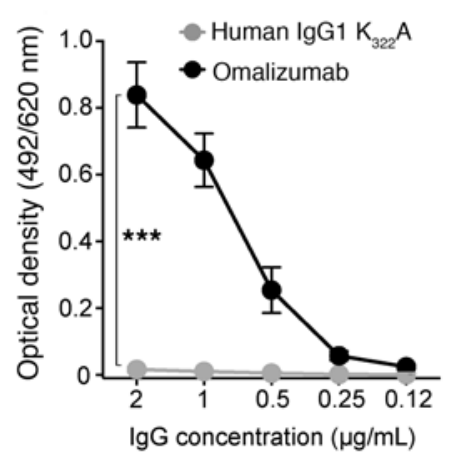

C

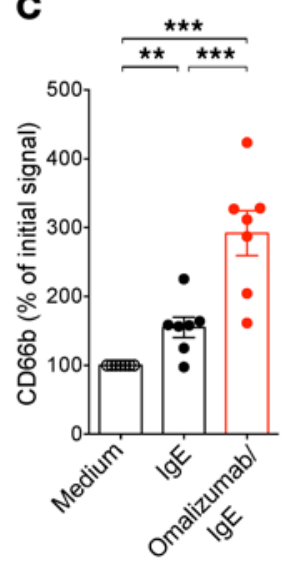

D

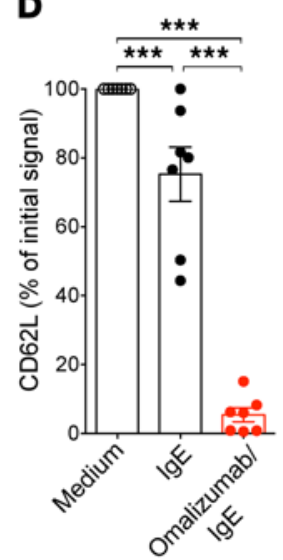

E

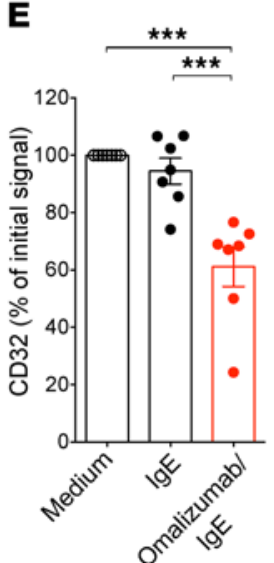

$\operatorname{lgE}$ FITC
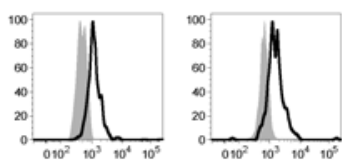

$\mathbf{F}$

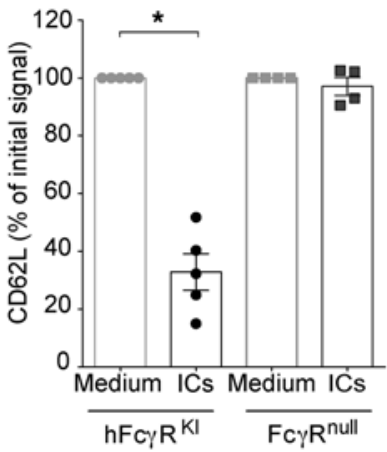

Figure 1. Omalizumab/IgE ICs bind FcyRs and activate neutrophils. (A) Binding of preformed IgE/omalizumab ICs to Fc $\gamma$ Rs in CHO cells stably transfected with each one of the human Fc $\gamma$ Rs (16). Upper histograms show binding of an anti-FLAG mAb as a control for Fc $\gamma R$ expression. Lower histograms show binding of ICs or IgE FITC alone. Data are representative of 3 independent experiments. (B) Binding of omalizumab to human C1q assessed by ELISA. An irrelevant IgG1 mutated in its Fc portion at position $322\left(\mathrm{~K}_{322} \mathrm{~A}\right)$ to preclude binding to $\mathrm{C} 1 \mathrm{q}$ was used as a negative control. Results in B show mean \pm SD from data pooled from 2 independent experiments (total of $n=4$ replicates). Expression of CD66b (C), CD62L (D), and CD32 (E) on purified CD45 ${ }^{+}$CD15 ${ }^{+}$ human neutrophils after 1 hour of incubation with omalizumab/IgE immobilized ICs, IgE, or medium alone. Results in C-E show values from neutrophils from individual donors normalized against cells stimulated with medium alone; bars indicate mean \pm SEM of $n=7$ total values per group pooled from 3 independent experiments. (F) CD62L expression on CD11 ${ }^{+}$Ly6 $6 \mathrm{C}^{+}$neutrophils purified form $\mathrm{hF} \gamma \mathrm{R} \mathrm{R}^{\mathrm{KI}}$ or Fc $\gamma \mathrm{R}^{\text {null }}$ mice after 1 hour of incubation with ICs or medium. Results in $\mathbf{F}$ show values from individual mice with bars indicating mean $\pm \mathrm{SEM}$ pooled from 2 ( $F c \gamma \mathrm{R}^{\text {null; }}$ t total $n=4 / \mathrm{group}$ ) or 3 ( $\mathrm{hFc} \gamma \mathrm{R}^{\mathrm{Kl}}$; total $n=5$ /group) independent experiments. ${ }^{*} P<0.05$; ${ }^{* *} P<0.01$; ${ }^{* *} P<0.001$ using 1-way ANOVA in $\mathbf{B}$, contrast linear model in $\mathbf{C}-\mathbf{E}$, and Welch test in $\mathbf{F}$. For further details on the statistical analysis, please refer to Supplemental Table 1.

As neutrophils were reported to contribute to IgG-mediated inflammation and anaphylaxis (17), we next evaluated whether omalizumab/IgE ICs can activate neutrophils through engagement of Fc $\gamma$ Rs. We purified neutrophils from healthy donors and incubated these cells with omalizumab/IgE ICs. We found that such ICs induce marked upregulation of $\mathrm{CD} 66 \mathrm{~b}$ and downregulation of CD62L on the surface of neutrophils, which are considered hallmarks of neutrophil activation $(18,19)$ (Figure 1, C and D). The ICs also induced downregulation of Fc $\gamma$ RII (CD32) (Figure 1E). As human neutrophils express Fc $\gamma$ RIIA and not Fc $\gamma$ RIIB (20), and omalizumab/IgE ICs do not bind Fc $\gamma$ RIIB (Figure 1A), our results indicate that the ICs induce active engagement of Fc $\gamma$ RIIA on neutrophils.

To further confirm the role of Fc $\gamma$ Rs in neutrophil activation, we performed similar experiments with neutrophils purified from $\mathrm{hFc} \gamma \mathrm{R}^{\mathrm{KI}}$ mice (in which all mouse $\mathrm{Fc} \gamma \mathrm{Rs}$ have been replaced with human Fc $\gamma R s$ ) or Fc $\gamma R^{\text {null }}$ mice (deficient for all $F c \gamma R s$ ) (Figure $1 F$ ) (21). Omalizumab/IgE ICs induced a downregulation of CD62L in neutrophils from $\mathrm{hFc} \gamma \mathrm{R}^{\mathrm{KI}}$ mice, but not in neutrophils from $\mathrm{Fc} \gamma \mathrm{R}^{\text {null }}$ mice (Figure 1F), demonstrating that omalizumab/IgE can activate neutrophils through engagement of human $\mathrm{Fc} \gamma \mathrm{Rs}$.

The most frequent side effect observed with omalizumab is skin inflammation (13). We hypothesized that such local inflammation could be a consequence of Fc $\gamma$ Rs engagement. To assess this, we injected omalizumab/IgE ICs subcutaneously into hairless (to avoid shaving-induced skin inflammation) nude $\mathrm{hFc} \gamma \mathrm{R}^{\mathrm{KI}}$ mice and nude $\mathrm{Fc} \gamma \mathrm{R}^{\text {null }}$ mice, and assessed skin inflammation after 2 hours by bioluminescence imaging of myeloperoxidase (MPO) activity $(20,22)$. We observed strong MPO activity at the site of IC injection in $\mathrm{hFc} \gamma \mathrm{R}^{\mathrm{KI}}$ mice (Figure 2, A and B). By contrast, $\mathrm{MPO}$ activity was markedly reduced upon injection of $\operatorname{IgE}$ alone or omalizumab alone, or injection of ICs in Fc $\gamma \mathrm{R}^{\text {null }}$ mice. Thus, our results indicate that omalizumab/IgE ICs can induce skin inflammation through engagement of hFc $\gamma$ Rs.

The most dramatic side effect reported for omalizumab is anaphylaxis $(12,13)$. We thus assessed whether omalizumab/ IgE ICs can induce anaphylaxis in $\mathrm{hFc} \gamma \mathrm{R}^{\mathrm{KI}}$ mice. Intravenous 
A

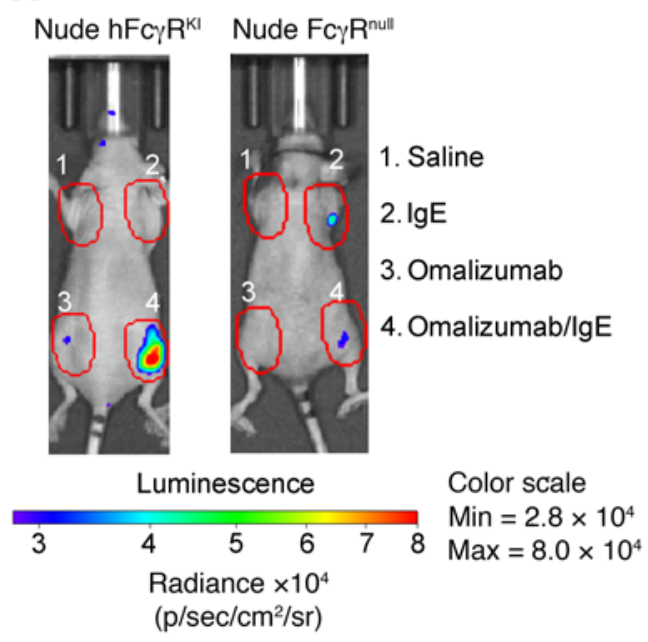

B

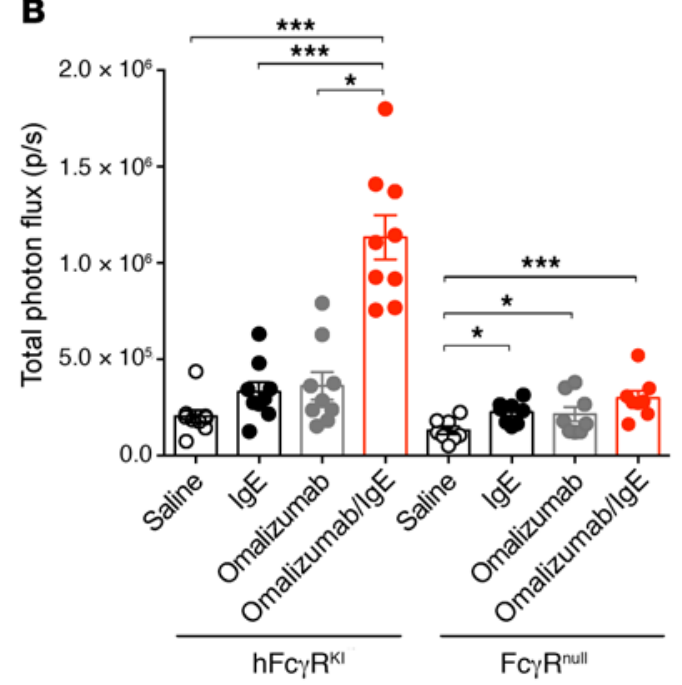

D

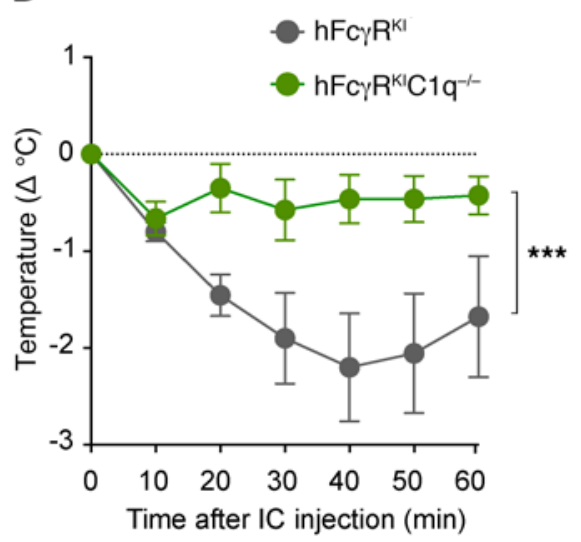

Figure 2. Omalizumab/IgE ICs induce skin inflammation and anaphylaxis through engagement of FcyRs in FcyRhumanized mice. Representative bioluminescent images (A) and quantification (B) of MPO activity 2 hours after subcutaneous injection of IgE/omalizumab ICs in nude hFc $\gamma \mathrm{R}^{\mathrm{KI}}$ mice $(n=$ 9) or nude Fc $\gamma \mathrm{R}^{\text {null }}$ mice $(n=8)$. Regions of interest outlined in red in $\mathbf{A}$ surround sites of injection. Data in $\mathbf{B}$ are mean \pm SEM pooled from 2 independent experiments. (C and $\mathbf{D})$ Changes in body temperature $\left(\triangle^{\circ} \mathrm{C}\right.$ [mean \pm SEM]) after intravenous injection of IgE/omalizumab ICs into $\mathrm{hFc} \gamma \mathrm{R}^{\mathrm{KI}}$ mice $(n=13)$ or $\mathrm{Fc} \gamma \mathrm{R}^{\text {null }}$ mice $(n=9)(C)$, or hFc $\gamma R^{\mathrm{KI}}$ mice $(n=9)$ or $\mathrm{hFc} \gamma \mathrm{R}^{\mathrm{Kl}} \mathrm{Cl}^{-1-}$ mice $(n=$ 8). Data are pooled from 3 (C) or 2 (D) independent experiments. ${ }^{*} P$ $<0.05 ;{ }^{* *} P<0.001$ by contrast test in linear model (B and C) or ANOVA (D). For additional details on the statistical analysis, please refer to Supplemental Table 1. injection of ICs induced significant hypothermia (the main readout of anaphylaxis in mice, ref. 23) in $\mathrm{hFc} \mathrm{R}^{\mathrm{KI}}$ mice (Figure $2 \mathrm{C}$ ). Importantly, hypothermia was not observed upon IC injection in $\mathrm{Fc} \gamma \mathrm{R}^{\text {null }}$ mice (Figure 2C) or injection of IgE or omalizumab alone in $\mathrm{hFc} \gamma \mathrm{R}^{\mathrm{KI}}$ mice (Supplemental Figure 2), demonstrating that the ICs induce systemic anaphylaxis through engagement of human Fc $\gamma$ Rs. Previous work indicates that hFc $\gamma$ RIIA contributes to experimental anaphylaxis in humanized mice $(21,24,25)$. We did not observe anaphylaxis in $\mathrm{Fc} \mathrm{R}^{\text {null }}$ mice transgenic for hFc $\gamma$ RIIA (which express only hFc $\gamma$ RIIA, ref. 21), indicating that hFc $\gamma$ RIIA is not sufficient to trigger omalizumab/IgE-mediated anaphylaxis (Supplemental Figure 3A). By contrast, anaphylaxis was markedly reduced in $\mathrm{hFc} \gamma \mathrm{R}^{\mathrm{KI}}$ mice pretreated with a blocking mAb against hFc $\gamma$ RIII (Supplemental Figure 3B). This suggests that hFc $\gamma$ RIII plays an important role in omalizumab/IgEmediated anaphylaxis. These results have to be interpreted carefully, as we cannot exclude that pretreatment with the anti$h F c \gamma R I I I m A b$ induces engagement of the receptor to some extent, thereby desensitizing cells expressing hFc $\gamma$ RIII.

Since omalizumab also binds complement component $\mathrm{C} 1 \mathrm{q}$ (Figure 1B), we assessed the potential contribution of $\mathrm{C} 1 \mathrm{q}$ to IC-induced anaphylaxis. We found that anaphylaxis is markedly reduced in $\mathrm{hFc} \gamma \mathrm{R}^{\mathrm{KI}} \mathrm{Cl} \mathrm{q}^{-/-}$mice, which express all $\mathrm{hFc} \gamma \mathrm{Rs}$ but lack mouse $\mathrm{C} 1 \mathrm{q}$ (Figure 2D). Although further work is required to con- firm the implication of human C1q, our data strongly suggest that the complement pathway plays an important role, through $\mathrm{C} 1 \mathrm{q}$ engagement, in omalizumab/IgE-induced anaphylaxis.

Based on these results, we decided to produce an Fc-engineered form of omalizumab (using available omalizumab $\mathrm{V}_{\mathrm{H}}$ and $\mathrm{V}_{\mathrm{L}}$ sequences, ref. 4) lacking the $\mathrm{N}$-linked glycan attached to asparagine 297 in the $\mathrm{Fc}$ portion $\left(\mathrm{N}_{297} \mathrm{~A}\right.$ mutation) to reduce binding to Fc $\gamma$ Rs and complement $(20,26)$. We refer to this mAb as NA antiIgE. As a control, we also produced a nonmutated version of this $\mathrm{mAb}$ (WT anti-IgE). Both the WT and NA anti-IgE mainly formed trimers when incubated with IgE in vitro (Supplemental Figure 4, $\mathrm{A}-\mathrm{D})$, which is consistent with the data we obtained using commercial omalizumab (Supplemental Figure 1). As expected, ICs made of IgE and the WT anti-IgE could bind all activating Fc $\gamma$ Rs, whereas binding to Fc $\gamma$ Rs was markedly reduced with ICs made of IgE and the NA anti-IgE (Figure 3A). Indeed, IgE/NA anti-IgE ICs could only bind to Fc $\gamma$ RI, which is consistent with a previous report showing that the $\mathrm{N}_{297}$ A mutation does not abrogate binding to this high-affinity Fc $\gamma \mathrm{R}$ (27). In addition, WT anti-IgE could bind human C1q (Supplemental Figure 4E), but we detected no binding to C1q with the NA anti-IgE (Supplemental Figure 4E). Finally, we observed activation of human neutrophils with ICs made of IgE and the WT anti-IgE, but markedly reduced activation with ICs made of IgE and the NA anti-IgE (Figure 3, B-D). 
A
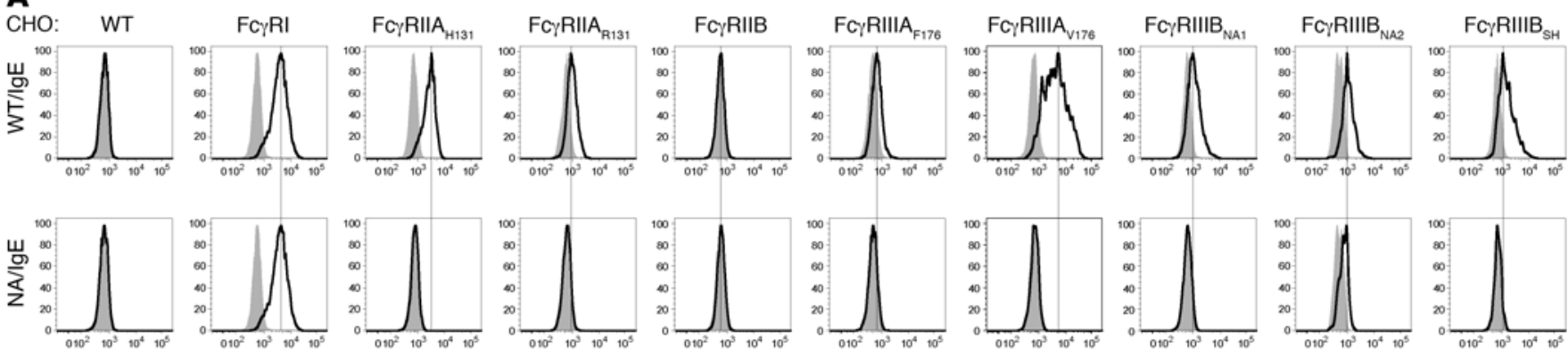

FITC

B

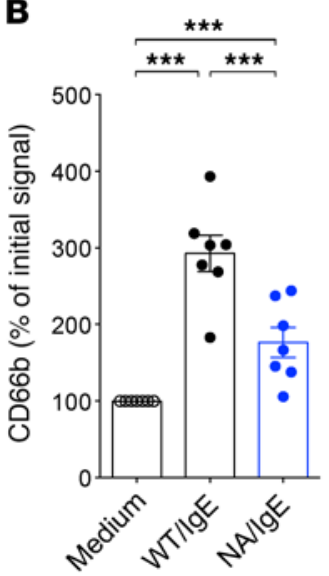

C

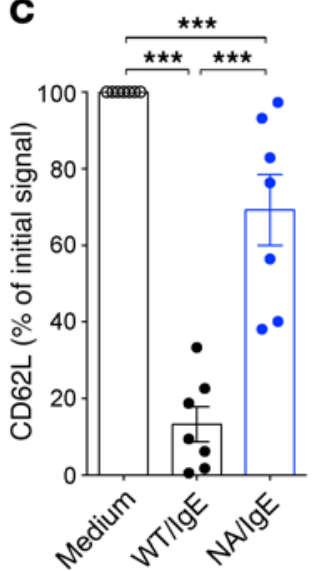

D

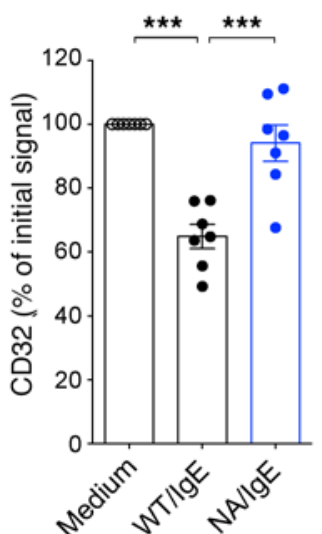

$\lg$ E FITC

Figure 3. Fc-engineered anti-IgE antibodies display markedly reduced Fc $\gamma$ R-binding and neutrophil activation. (A) Binding of ICs made of FITC-IgE and WT anti-IgE or Fc-engineered N297A (NA) anti-IgE. Data are representative of 3 independent experiments. Expression of CD66b (B), CD62L (C), and CD32 (D) on purified CD45+CD15+ human neutrophils after 1 hour of incubation with IgE/WT anti-lgE or IgE/NA anti-IgE ICs or medium alone. Results in B-D show values from neutrophils from individual donors normalized against cells stimulated with medium alone. Bars indicate mean \pm SEM pooled from 3 independent experiments (total $n=7 /$ group). (E) $100 \mu \mathrm{g}$ of WT or NA anti-IgE was injected i.p. into hFc $\gamma \mathrm{R}^{\mathrm{K} l} \mathrm{hFcR} n^{\mathrm{K}} \mathrm{h} \beta 2 \mathrm{~m}^{\mathrm{Kl}}$ mice, and serum was collected at different time points. Levels of anti-IgE mAbs were measured by ELISA. Data are indicated as mean \pm SEM pooled from 2 independent experiments ( $n=13 /$ group). ${ }^{* * *} P<$ 0.001 by contrast linear model in B-D and ANOVA in E. For additional details on the statistical analysis, please refer to Supplemental Table 1.

$\mathrm{FcRn} / \beta 2 \mathrm{~m}$ heterodimers extend the half-life of IgG by reducing lysosomal degradation in endothelial cells (28). To assess the half-life of our anti-IgE mAbs in vivo, we used $h F c \gamma \mathrm{R}^{\mathrm{K}} \mathrm{hFcRn}{ }^{\mathrm{KL}}$ $\mathrm{h} \beta 2 \mathrm{~m}^{\mathrm{KI}}$ mice, which recapitulate binding of IgG to all human Fc $\gamma \mathrm{Rs}$ and to the human FcRn- $\beta 2 \mathrm{~m}$ complex (Supplemental Figure 5) (29). We injected WT or NA anti-IgE into $h F c \gamma \mathrm{R}^{\mathrm{KI}} \mathrm{hFcRn}{ }^{\mathrm{K}} \mathrm{h} \beta 2 \mathrm{~m}^{\mathrm{KI}}$ mice, and observed similar mAb levels in sera collected at different time points (Figure 3E). We obtained similar results when comparing the half-life of commercial omalizumab and the Fcengineered NA anti-IgE (Supplemental Figure 6). Altogether, these results demonstrate that the $\mathrm{N}_{297} \mathrm{~A}$ mutation does not affect the half-life of the anti-IgE $\mathrm{mAb}$ in vivo.

We also verified that the $\mathrm{N}_{297} \mathrm{~A}$ mutation does not affect the ability of the anti-IgE mAb to block IgE. Both the WT and NA antiIgE recognized IgE with the same affinity (Supplemental Figure $7 \mathrm{~A})$, and were equally potent at blocking binding of IgE to human mast cells (Supplemental Figure 7B). Moreover, we showed that pretreatment of $\mathrm{hFc} \varepsilon \mathrm{RI}^{\mathrm{Tg}}$ mice (which express the human IgE receptor hFc\&RI, ref. 30) with either omalizumab or the NA anti-IgE can block IgE-mediated anaphylaxis (Figure 4A). Altogether, our results demonstrate that the $\mathrm{Fc}$-engineered $\mathrm{NA}$ anti-IgE is equally potent as omalizumab at blocking IgE-mediated allergic reactions.
We then compared skin inflammation induced by IgE/omalizumab or IgE/NA anti-IgE ICs in $\mathrm{hFc} \gamma \mathrm{R}^{\mathrm{KI}}$ mice. Injection of $\mathrm{IgE} /$ omalizumab ICs induced marked MPO activity in the skin (Figure 4, $B$ and $C$ ). This was reduced to levels observed with injection of IgE alone in $\mathrm{hFc} \gamma \mathrm{R}^{\mathrm{KI}}$ mice injected with IgE/NA anti-IgE ICs (Figure 4, B and C). Finally, we compared the ability of ICs made of IgE and omalizumab or the NA anti-IgE to induce anaphylaxis in $\mathrm{hFc} \gamma \mathrm{R}^{\mathrm{KI}}$ mice. We observed anaphylaxis in mice injected with IgE/omalizumab ICs but not in mice injected with IgE/NA anti-IgE ICs (Figure 4D).

In summary, our findings demonstrate that omalizumab forms ICs with IgE, which can activate neutrophils and induce skin inflammation and systemic anaphylaxis through human Fc $\gamma$ Rs in Fc $\gamma$ R-humanized mice. Such findings could explain some of the side effects that have been described in patients treated with omalizumab $(12,13)$. One must be careful when extrapolating these findings obtained in humanized mice to humans, as very few data have been reported supporting the existence of Fc $\gamma \mathrm{R}$-mediated anaphylaxis in humans. However, one recent report provides evidence of an IgG-induced, Fc $\gamma$ R-dependent neutrophil activation pathway in anaphylaxis to neuromuscular-blocking agents (NMBAs) in humans (31), which reinforces the potential clinical relevance of our findings. The Fc-engineered anti-IgE $\mathrm{mAb}$ we 
A
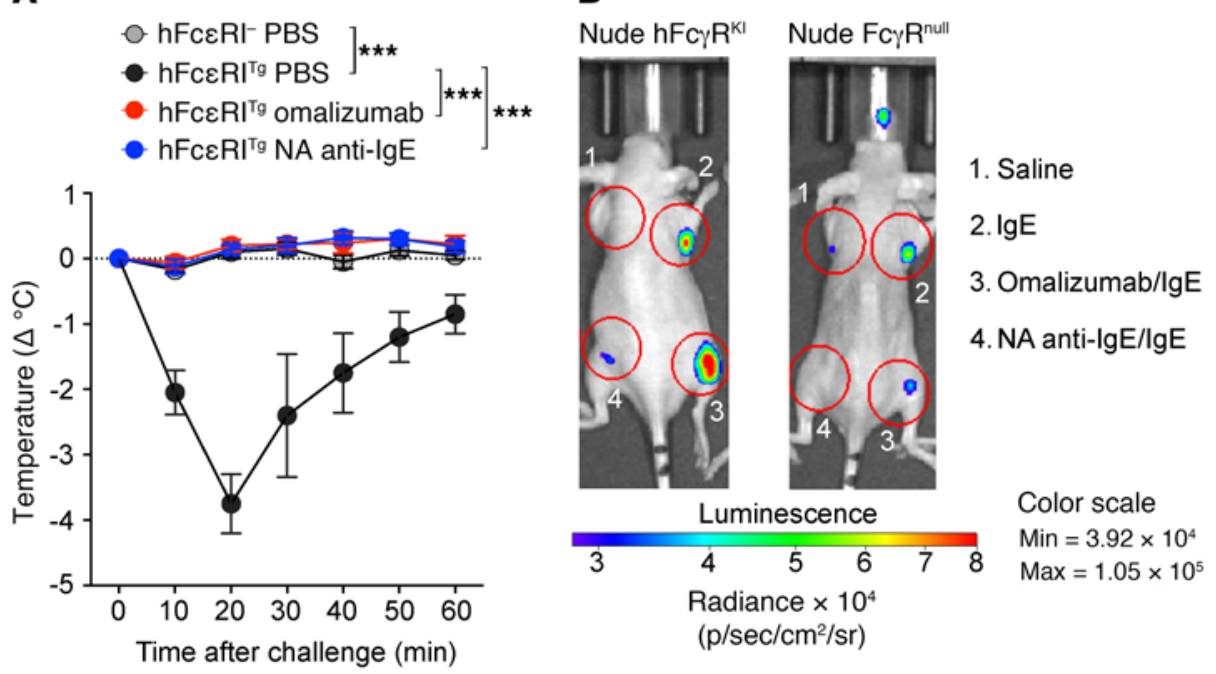

C

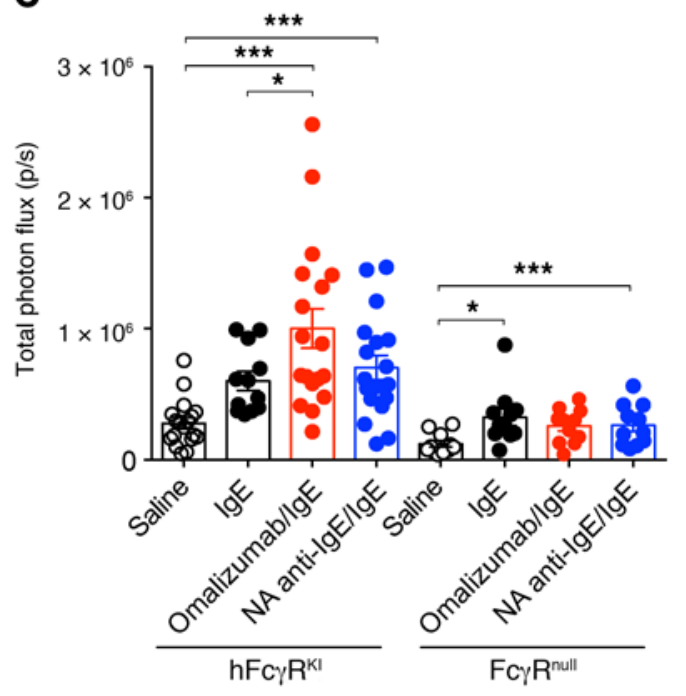

D Omalizumab/lgE

NA anti-IgE/lgE

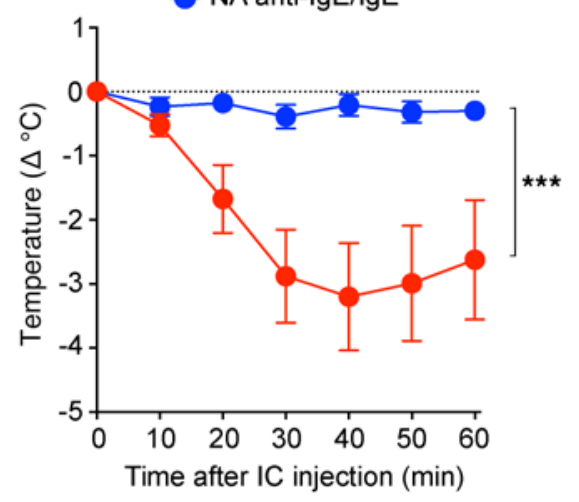

Figure 4. Fc-engineered anti-IgE antibodies block IgE-mediated anaphylaxis but do not induce FcyR-dependent inflammation. (A) Changes in body temperature $\left(\Delta^{\circ} \mathrm{C}\right.$ [mean $\pm \mathrm{SEM}]$ ) after i.v. challenge with $500 \mu \mathrm{g}$ nitrophenyl-coupled

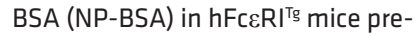
treated i.v. with $700 \mu$ g omalizumab NA anti-IgE, or PBS 30 minutes before sensitization with anti-NP IgE $(10 \mu \mathrm{g})$. Data in $\mathbf{A}$ are pooled from 2 independent experiments (total $n=$ 4-6/group). hFceRl-mice were used as a control. Representative bioluminescent images (B) and quantification (C) of MPO activity 2 hours after subcutaneous injection of IgE/ omalizumab or IgE/NA anti-IgE ICs in nude $\mathrm{hFc} \gamma \mathrm{R}^{\mathrm{Kl}}$ or nude $\mathrm{F} c \gamma \mathrm{R}^{\text {null }}$ mice. Regions of interest outlined in red surround the site of injection. Bars in C indicate mean \pm SEM pooled from 5 (nude hFc $\gamma \mathrm{R}^{\mathrm{KI}}$, total $n=18$ ) or 4 (nude Fc $\gamma \mathrm{R}^{\text {null }}$, total $n=11$ ) independent experiments. The color bar represents bioluminescent signal in radiance ( $\left.p / \mathrm{sec} / \mathrm{cm}^{2} / \mathrm{sr}\right)$. (D) Changes in body temperature $\left(\Delta^{\circ} \mathrm{C}\right.$ [mean \pm SEM]) after i.v. injection of IgE/ omalizumab $(n=10)$ or IgE/NA antiIgE $(n=11)$ into $h F c \gamma R^{\mathrm{KI}}$ mice. Data in $\mathbf{D}$ are pooled from 2 independent experiments. ${ }^{*} P<0.05 ;{ }^{* * *} P<0.001$ by contrast test linear model (A and $\mathbf{C}$ ) or 2-way repeated-measures (ANOVA) (D). For additional details on the statistical analysis, please refer to Supplemental Table 1. developed is equally potent as omalizumab at blocking IgE-mediated allergic reactions but does not induce Fc $\gamma \mathrm{R}$-mediated inflammation. It could thus potentially be used in patients with very high levels of $\operatorname{IgE}$ and/or in patients with a history of anaphylaxis or other adverse reactions to omalizumab. Finally, we envision that IC-mediated engagement of Fc $\gamma$ Rs could be a more general mechanism of therapeutic $\mathrm{mAb}$-mediated adverse reactions.

\section{Methods}

See the Supplemental Methods for the description of all experimental procedures and statistical analyses.

Study approval. All animal care and experimentation were conducted in compliance with the guidelines and specific approval of the Animal Ethics Committee CETEA (Institut Pasteur, Paris, France) registered under \#2013-0103, and by the French Ministry of Research under agreement 00513.02.

\section{Author contributions}

$\mathrm{BB}, \mathrm{PB}$, and LLR designed the experiments. BB, PH, OG, JS, and LLR conducted experiments. BB, PH, OG, JS, ORL, BI, DS, SB, and LLR acquired data. FMH, VAV, LEM, and AJM provided mice. KCN provided reagents. GAM performed statistical analysis. BB and LLR conducted the formal analysis. BB and LLR wrote the original draft of the manuscript. All authors contributed to review and editing of the manuscript.

\section{Acknowledgments}

This work was supported by the European Commission (Marie Skłodowska-Curie Individual Fellowship H2020-MSCA-IF-2014 656086 to LLR) and the European Research Council (ERC) Seventh Framework Program (ERC-2013-CoG 616050 to PB), the Institut Pasteur initiative for valorizing the applications of research (ValoExpress 2017), and the Institut National de la Santé et de la Recherche Médicale (INSERM) and ATIP-Avenir program (to LLR). Part of this work was performed on a platform member of France Life Imaging network, partly funded by the French program "Investissement d'Avenir" (grant ANR-11-INBS-0006). BB was supported partly by a stipend from the Pasteur -Paris University (PPU) International PhD program and a fellowship from the French "Fondation pour la Recherche Médicale FRM". DS bene- 
fited from a stipend ("Poste d'accueil") provided by AP-HP, Paris, France, and by the Institut Pasteur, Paris, France.

Address correspondence to: Laurent L. Reber, ATIP-Avenir team "Asthma, Allergy \& Immunotherapy," Center for Physiopathology
Toulouse-Purpan, CHU Purpan - BP 3028, 31024 Toulouse Cedex 3, France. Phone:33.5.6274.4529; Email: laurent.reber@inserm.fr. Or to: Pierre Bruhns, Unit of Antibodies in Therapy and Pathology, Department of Immunology, Institut Pasteur, 25 rue du Docteur Roux, Paris, 75015, France.Phone:33.1.4568.8629; Email: bruhns@pasteur.fr.
1. Gould HJ, Sutton BJ. IgE in allergy and asthma today. Nat Rev Immunol. 2008;8(3):205-217.

2. Balbino B, Conde E, Marichal T, Starkl P, Reber LL. Approaches to target IgE antibodies in allergic diseases. Pharmacol Ther. 2018;191:50-64.

3. Galli SJ, Tsai M. IgE and mast cells in allergic disease. Nat Med. 2012;18(5):693-704.

4. Presta LG, et al. Humanization of an antibody directed against IgE. JImmunol. 1993;151(5):2623-2632.

5. Chang TW, Davis FM, Sun NC, Sun CR, MacGlashan DW, Hamilton RG. Monoclonal antibodies specific for human IgE-producing B cells: a potential therapeutic for IgE-mediated allergic diseases. Biotechnology (NY). 1990;8(2):122-126.

6. Selb R, et al. Critical and direct involvement of the CD23 stalk region in IgE binding. J Allergy Clin Immunol. 2017;139(1):281-289.e5.

7. Davies AM, et al. Allosteric mechanism of action of the therapeutic anti-IgE antibody omalizumab. J Biol Chem. 2017;292(24):9975-9987.

8. Busse W, et al. Omalizumab, anti-IgE recombinant humanized monoclonal antibody, for the treatment of severe allergic asthma. J Allergy Clin Immunol. 2001;108(2):184-190.

9. Maurer M, et al. Omalizumab for the treatment of chronic idiopathic or spontaneous urticaria. N Engl JMed. 2013;368(10):924-935.

10. MacGinnitie AJ, et al. Omalizumab facilitates rapid oral desensitization for peanut allergy. JAllergy Clin Immunol. 2017;139(3):873-881.e8.

11. Limb SL, Starke PR, Lee CE, Chowdhury BA. Delayed onset and protracted progression of anaphylaxis after omalizumab administration in patients with asthma. JAllergy Clin Immunol. 2007;120(6):1378-1381.

12. Lieberman PL, Umetsu DT, Carrigan GJ, Rahmaoui A. Anaphylactic reactions associated with omalizumab administration: analysis of a case-control study. J Allergy Clin Immunol. 2016;138(3):913-915.e2.

13. Lieberman PL, Jones I, Rajwanshi R, Rosén $\mathrm{K}$, Umetsu DT. Anaphylaxis associated with omalizumab administration: risk factors and patient characteristics. J Allergy Clin Immunol. 2017;140(6):1734-1736.e4.

14. Liu J, Lester P, Builder S, Shire SJ. Characterization of complex formation by humanized anti-IgE monoclonal antibody and monoclonal human IgE. Biochemistry. 1995;34(33):10474-10482.

15. Fox JA, Hotaling TE, Struble C, Ruppel J, Bates DJ, Schoenhoff MB. Tissue distribution and complex formation with IgE of an anti-IgE antibody after intravenous administration in cynomolgus monkeys. JPharmacol Exp Ther. 1996;279(2):1000-1008.

16. Bruhns $P$, et al. Specificity and affinity of human Fcgamma receptors and their polymorphic variants for human IgG subclasses. Blood. 2009;113(16):3716-3725.

17. Jönsson F, Mancardi DA, Albanesi M, Bruhns P. Neutrophils in local and systemic antibodydependent inflammatory and anaphylactic reactions. J Leukoc Biol. 2013;94(4):643-656.

18. Zhao L, Xu S, Fjaertoft G, Pauksen K, Håkansson $\mathrm{L}$, Venge P. An enzyme-linked immunosorbent assay for human carcinoembryonic antigenrelated cell adhesion molecule 8 , a biological marker of granulocyte activities in vivo. J Immunol Methods. 2004;293(1-2):207-214.

19. Pillay J, et al. A subset of neutrophils in human systemic inflammation inhibits T cell responses through Mac-1. J Clin Invest. 2012;122(1):327-336.

20. Bruhns P, Jönsson F. Mouse and human FcR effector functions. Immunol Rev. 2015;268(1):25-51.

21. Beutier $\mathrm{H}$, et al. Platelets expressing IgG recep- tor Fc $\gamma$ RIIA/CD32A determine the severity of experimental anaphylaxis. Sci Immunol. 2018;3(22): eaan5997.

22. Gross S, et al. Bioluminescence imaging of myeloperoxidase activity in vivo. Nat Med. 2009;15(4):455-461.

23. Strait RT, Morris SC, Yang M, Qu XW, Finkelman FD. Pathways of anaphylaxis in the mouse. JAllergy Clin Immunol. 2002;109(4):658-668.

24. Gillis CM, et al. Mechanisms of anaphylaxis in human low-affinity IgG receptor locus knock-in mice. JAllergy Clin Immunol. 2017;139(4):1253-1265.e14.

25. Jönsson F, et al. Human Fc $\gamma$ RIIA induces anaphylactic and allergic reactions. Blood. 2012;119(11):2533-2544.

26. Smith P, DiLillo DJ, Bournazos S, Li F, Ravetch JV. Mouse model recapitulating human $\mathrm{Fc} \gamma$ receptor structural and functional diversity. Proc Natl Acad Sci U S A. 2012;109(16):6181-6186.

27. Nesspor TC, Raju TS, Chin CN, Vafa O, Brezski RJ. Avidity confers Fc $\gamma \mathrm{R}$ binding and immune effector function to aglycosylated immunoglobulin G1. J Mol Recognit. 2012;25(3):147-154.

28. Stapleton NM, Einarsdóttir HK, Stemerding AM, Vidarsson G. The multiple facets of FcRn in immunity. Immunol Rev. 2015;268(1):253-268.

29. Lee CH, Kang TH, Godon O, et al. An engineered human Fc domain that behaves like a pH-toggle switch for ultra-long circulation persistence. Nat Commun. 2019;10(1):5031.

30. Dombrowicz D, et al. Anaphylaxis mediated through a humanized high affinity IgE receptor. Jimmunol. 1996;157(4):1645-1651.

31. Jönsson F, et al. An IgG-induced neutrophil activation pathway contributes to human drug-induced anaphylaxis. Sci Transl Med. 2019;11(500):eaat1479. 